\title{
A Facial Symmetry Prior for Improved Illumination Fitting of 3D Morphable Model
}

\author{
Guosheng Hu, Pouria Mortazavian, Josef Kittler, William Christmas \\ Centre for Vision, Speech and Signal Processing \\ University of Surrey, Guildford, UK

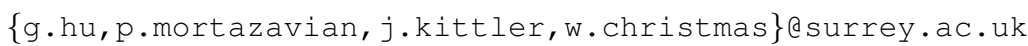

\begin{abstract}
$3 D$ face reconstruction from a single $2 D$ image can be performed using a 3D Morphable Model (3DMM) in an analysis-by-synthesis approach. However, the reconstruction is an ill-posed problem. The recovery of the illumination characteristics of the $2 D$ input image is particularly difficult because the proportion of the albedo and shading contributions in a pixel intensity is ambiguous. In this paper we propose the use of a facial symmetry constraint, which helps to identify the relative contributions of albedo and shading. The facial symmetry constraint is incorporated in a multi-feature optimisation framework, which realises the fitting process. By virtue of this constraint better illumination parameters can be recovered, and as a result the estimated $3 D$ face shape and surface texture are more accurate. The proposed method is validated on the PIE face database. The experimental results show that the introduction of facial symmetry constraint improves the performance of both, face reconstruction and face recognition.
\end{abstract}

\section{Introduction}

The 3D morphable model (3DMM) is a well-known tool for 3D face reconstruction from a single 2D input image $[1,2]$. Once the 3D model is fitted to the input image, its main application is to synthesize other $2 \mathrm{D}$ face images of the same subject in different poses, acquisition conditions, and with different expression. The fitted 3DMM can also be used for face recognition, facial animation and face tracking [11]. By virtue of the fitting process, 3DMM estimates the 3D shape, texture, 3D pose and the light direction from a single input image. However this estimation process is an ill-posed problem, as we attempt to reconstruct the parameters that have been lost through the projection from $3 \mathrm{D}$ into $2 \mathrm{D}$. As a result the reconstruction process can easily be derailed by noise, spurious illumination conditions, or unfavourable initialisation. The $3 \mathrm{D}$ face model fitting is therefore a very challenging problem. Moreover, most optimisation strategies are based on iterative gradient descent, which is computationally intensive and easily trapped in local minima, thus reducing the fitting accuracy.

The original 3DMM optimisation algorithm $[1,2] \mathrm{min}-$ imises the sum of square differences over all color channels and all pixels between the input image and the reconstructed image. Gaussian prior probability distributions over shape and texture respectively are used to regularise the cost function in order to avoid over-fitting. A stochastic Newton optimisation (SNO) was developed to optimise the cost function. Not surprisingly, the performance in terms of efficiency and accuracy of this first version of the 3DMM fitting process was not all that good.

Motivated by the above challenges, considerable research has been carried out to improve the fitting process. One attempt at improving the efficiency of the optimisation is the Inverse Compositional Image Alignment (ICIA) algorithm [7, 13]. ICIA was first developed to optimise Active Appearance Models(AAMs)[3]. In [13] it was extended to optimise 3DMM fitting by modifying the cost function in which the Jacobian matrix could be regarded as constant. Consequently the Jacobian matrix did not need to be updated at each iteration. Clearly, this improved the optimisation efficiency greatly. However, as ICIA was developed for AAMs, which only modeled shape and texture rather than the imaging phenomena, it could not optimise illumination properly, which limited its performance.

Other enhancements based on ICIA include a multiresolution fitting strategy [5]. The optimisation was first conducted on a low resolution 3DMM. The estimated parameters were then used to initialise a medium resolution model. The result of fitting initialised the high resolution model to obtain the final fitting results. A Similar multi-resolution idea was also proposed by [4]. This multiresolution fitting reduces the probability of getting stuck in a local optimum during the fitting process.

The efficiency of optimisation is the driver behind a linear shape and texture fitting algorithm (LiST) [12]. The 
basic idea of LiST is to update the gradients of the shape and texture parameters by solving a linear system. The objective is to convert the non-linear optimisation problem to a linear optimisation. The illumination and rigid transformation parameters are optimised by Levenberg-Marquardt method because this aspect of the fitting problem cannot be converted to a linear system. The experiments reported in [12] showed that the fitting is 5 times faster than with the SNO algorithm. However, in this approach it is assumed that light direction is known before fitting, which is not realistic for automatic optimisation. Another drawback is that the texture information is not used for the shape parameter estimation. This may affect the quality of the result of optimisation.

To the best of our knowledge, the Multi-Feature Fitting (MFF) [11] is the most successful fitting strategy so far in terms of accuracy. MFF makes use of complementary features extracted from the input image to constrain the fitting process. Besides the features used by $[1,2]$, the multifeature fitting exploits the face texture edge, contour edge, specular highlight and texture constraint features. These features lead to a better solution as demonstrated by [11]. Based on this framework, [8] proposed some other multiresolution features to adapt the framework to low resolution image fitting.

The MFF approach introduces features that are more illumination invariant to improve the illumination fitting. However, it is not robust enough to recover the illumination parameters in all conditions. If the input image is acquired in strong side illumination, it is hard to reconstruct it with the correct illumination. An alternative, or rather supplementary strategy to MFF is to introduce prior knowledge. Based on the fact that human face is roughly symmetric, we propose the use of a facial symmetry constrain to control the fitting process in order to improve the accuracy of the estimated illumination parameters. The facial symmetry constraint helps the model to separate the texture and illumination contribution from the face image, which is one of the central problems during the fitting. The experimental results obtained with the proposed algorithm on the PIE database show that our facial symmetry feature improves the performance of both face reconstruction and face recognition.

The paper is organised as follows. In the next section we present a brief introduction of the 3D face morphable model. The novel fitting methodology that incorporates the symmetry constraint is introduced in Section 3. The proposed algorithms are evaluated in Section 4. Section 5 discusses the results obtained and draws the paper to a conclusion.

\section{3D morphable model}

The 3D Morphable Model (3DMM) was proposed by Blanz and Vetter [2]. It is a parametric model based on a vector space representation of faces. In our implementation the model is constructed from a database of 3D face scans registered using Iterative Multi-Resolution Dense 3D Registration (IMDR) algorithm [10]. The information of shape and texture is conveyed by:

$$
\begin{aligned}
& \mathbf{s}=\left(x_{1}, y_{1}, z_{1}, \ldots, x_{N}, y_{N}, z_{N}\right) \\
& \mathbf{t}=\left(r_{1}, g_{1}, b_{1}, \ldots, r_{N}, g_{N}, b_{N}\right)
\end{aligned}
$$

where $\left(x_{i}, y_{i}, z_{i}\right)$ are the $3 \mathrm{D}$ coordinates of the $i$ th vertex and $\left(r_{i}, g_{i}, b_{i}\right)$ are the RGB values at this $i$ th vertex. The 3D face is represented by $N$ vertices. Because the raw face data is highly redundant, PCA is performed separately on shape and texture vectors to decorrelate the data. Using the decorrelated coefficients, face shape and textures can be represented as

$$
\mathbf{s}=\overline{\mathbf{s}}+\sum_{i=1}^{m-1} \alpha_{i} \cdot \mathbf{s}_{i}, \quad \mathbf{t}=\overline{\mathbf{t}}+\sum_{i=1}^{m-1} \beta_{i} \cdot \mathbf{t}_{i}
$$

where $\overline{\mathbf{s}}$ and $\overline{\mathbf{t}}$ are the mean of shape and texture, respectively. $\mathbf{s}_{i}$ and $\mathbf{t}_{i}$ are the $i$ th eigenvectors of shape and texture [2]. Different $\alpha=\left[\alpha_{1}, \ldots \alpha_{m-1}\right]$ and $\beta=\left[\beta_{1}, \ldots \beta_{m-1}\right]$ determine different $\mathbf{s}$ and $\mathbf{t}$. The probability density function of $\boldsymbol{\alpha}$ is given by

$$
p(\alpha) \sim \exp \left[-\frac{1}{2} \sum_{i=1}^{m-1}\left(\alpha_{i} / \sigma_{i}\right)^{2}\right]
$$

where $\sigma_{i}^{2}$ are the eigenvalues of the shape covariance matrix. The probability density function of $p(\beta)$ can be specified similarly. During the fitting process $\alpha$ and $\beta$ are the main parameters to be optimised.

Once the 3D morphable model is constructed, the model can be used for fitting a given 2D image. The primary goal of fitting is to minimise the sum of square differences over all colour channels and all pixels between the synthesized image and the input image,

$$
E_{I}=\sum_{x, y}\left\|t_{i}(x, y)-t_{m}(x, y)\right\|^{2}
$$

where $E_{I}$ is the cost function. $t_{i}(x, y)$ is the intensity of input image at pixel $(x, y) . t_{m}(x, y)$ is the model's texture value projected from $3 \mathrm{D}$ space to $2 \mathrm{D}$ image coordinates $(\mathrm{x}, \mathrm{y})[10]$.

\subsection{Illumination and colour transform}

As this work focuses on estimating illumination during the fitting, we first introduce the details of the illumination model. 
The illumination is modeled by the Phong reflection model [2]. The Phong reflection model is an empirical model of local illumination. It describes the way a surface reflects light as a combination of ambient reflection, diffuse reflection and specular reflection.

Specifically, according to this imaging model, for each vertex, the red channel observation is given by

$$
l_{r}=t_{m, r}\left(l_{r, a m b}+l_{r, d i r}<\mathbf{n}, \mathbf{l}>\right)+k_{s}<\mathbf{v}, \mathbf{r}>^{\gamma} l_{r, d i r}
$$

where $t_{m, r}$ is the red channel ambient and diffuse light reflection constant. It is stored in the texture vector $\mathbf{t}$ of Eq (1). Vector $\mathbf{n}$ denotes the surface norm. $\mathbf{l}$ is the direction of directed light, $\mathbf{v}$ is the viewing direction, $\mathbf{r}=2<\mathbf{n}, \mathbf{l}>\mathbf{n}-\mathbf{l}$ is the direction of maximum specular reflection, $k_{s}$ is the specular reflectance, $\gamma$ is a shininess constant for this material. $l_{r, a m b}$ is the strength of the ambient light, and $l_{r, d i r}$ is the strength of the directed light. The green and blue channels can be computed similarly.

The total luminance $l_{\text {all }}$ is defined by:

$$
l_{\text {all }}=0.3 l_{r}+0.59 l_{g}+0.11 l_{b}
$$

Colour contrast $c$ is introduced to interpolate between the original colour value and the total luminance. Accordingly, the colour value $m_{r}$ is defined by:

$$
m_{r}=c l_{r}+(1-c) l_{a l l}
$$

In order to extend the model to handle a variety of images such as colour images, grey-level images and even paintings, gains $g_{r}, g_{g}, g_{b}$ and offsets $o_{r}, o_{g}, o_{b}$ are applied to this model:

$$
i_{r}=g_{r} m_{r}+o_{r}
$$

Green and blue channels are computed in the same way. The colours $i_{r}, i_{g}$ and $i_{b}$ are drawn in the image plane.

\section{Methodology}

\subsection{Facial symmetry feature}

The proportion of the albedo and shading in the intensity of a pixel is ambiguous during the fitting process. An incorrect proportion will affect the fitting quality, in particular for input images under strong point light illumination. The injection of any prior knowledge pertaining to the general human facial texture into the illumination estimation process is likely to help with the resolution of the ambiguity.

Facial symmetry is a physical manifestation of human beings. Indeed, facial symmetry has been widely used for assisting face analysis [14,9] in computer vision. However, the human face is not perfectly symmetrical as quantified in [6]. In our work, we are not assuming that the human face is perfectly symmetrical. Our main assumption is that the difference caused by facial asymmetry is less than the difference caused by shading. Based on this assumption facial symmetry is introduced as a constraint to assist the fitting.

In the context of 3DMM, facial symmetry means the albedo contribution between the left and right facial part is the same. Specifically, the texture value of a vertex (from $t$ in $\mathrm{Eq}(1))$ in the left part of the face is the same as the corresponding vertex in the right part. Hence, the cost function incorporating facial symmetry can be written as:

$$
\begin{gathered}
C^{s}=\sum_{j=1}^{n}\left\|t_{l, j}-t_{r, j}\right\|^{2} \\
t_{l, j}=\bar{t}_{k 1}+\sum_{i=1}^{m-1} \beta_{i} \cdot t_{i, k 1} \\
t_{r, j}=\bar{t}_{k 2}+\sum_{i=1}^{m-1} \beta_{i} \cdot t_{i, k 2}
\end{gathered}
$$

where $t_{l, j}$ is the albedo intensity of the $j$ th vertex in left part and $t_{r, j}$ is the same for the corresponding vertex in the right part. $n$ is the number of vertices in one facial side. $k 1, k 2$ are the positions in $\mathbf{t}(\mathrm{Eq}(1))$ which correspond to the $j$ th vertex in left part and right part respectively. The derivative of $C^{s}$ with respect to $\beta_{i}$ is

$$
\frac{\partial C^{s}}{\partial \beta_{i}}=\sum_{k 1=k 2=1}^{n}\left(t_{i, k 1}-t_{i, k 2}\right)
$$

In total, $2 n$ vertices are used to construct the cost function. They do not move on the face surface as the pose changes. Also, they do not depend on the illumination. Hence, they can be regarded as a constant subset of model vertices. These symmetrical $2 n$ vertices were obtained offline. Specifically, the $n$ vertices on the left part were obtained manually, and the corresponding vertices on the right part are computed based on the symmetry relationship.

\subsection{Optimisation framework}

We incorporated the facial symmetry constraint in the Multi-Feature Fitting (MFF) optimisation framework. Levenberg-Marquardt was used as the optimisation method. The whole optimisation consists of 5 stages. It is actually a coarse-to-fine strategy. Stages 1 and 2 estimate the pose and shape roughly. Stage 3 estimates the illumination and texture. Stage 4 refines the whole optimisation. Stage 5 independently optimises four regions (eyes, nose, mouth and the rest) respectively. In order to improve the illumination parameters estimation, facial symmetry constraint is applied in Stage 3. The other four stages in our work are similar to [11]. The details of the 5 stages of the proposed method can be summarised as follows: 
In Stage 1, we roughly estimate the rigid transform parameters based on landmarks (anchor feature[11]). The cost function is:

$$
C_{1}=C^{a}
$$

where $C_{1}$ denotes the cost function of Stage $1, C^{a}$ denotes cost function for anchor feature.

In Stage 2, we estimate the rigid transform parameters and the first $20 \%$ of the shape coefficients with anchor landmarks, multi-resolution texture and contour edge features [8] and shape prior feature $(\mathrm{Eq}(2))$. Because edges are not robust, we use edge information to estimate only the first $20 \%$ shape coefficients. The cost function is:

$$
C_{2}=\omega_{1} C^{a}+\omega_{2} C^{t e}+\omega_{3} C^{c e}+\omega_{4} C^{s p}
$$

where $C^{t e}$ and $C^{c e}$ denote cost functions for multiresolution texture and contour edge respectively, $C^{s p}$ is the cost function for shape prior feature, and $\omega$ s are weighting constants.

In Stage 3, the illumination and the first $20 \%$ texture coefficients are optimised using pixel colour feature (the same as in Eq (3)), the proposed facial symmetry constraint and the texture prior feature $(\mathrm{Eq}(2))$. The cost function is:

$$
C_{3}=\omega_{1} C^{p}+\omega_{2} C^{s}+\omega_{3} C^{t p}
$$

where $C^{p}, C^{s}$ and $C^{t p}$ denote cost functions for pixel colour, facial symmetry and texture prior features, respectively.

In Stage 4, all the parameters (rigid transform, illumination, all the shape and texture coefficients) are optimised. The features used at this stage are pixel colour, multi-resolution contour edge, texture prior and shape prior. This stage refines all the estimated parameters of the first 3 stages. The cost function is:

$$
C_{4}=\omega_{1} C^{p}+\omega_{2} C^{c e}+\omega_{3} C^{t p}+\omega_{4} C^{s p}
$$

In Stage 5, we segment the face into 4 parts (eyes, nose, mouth and rest) and thus four groups of shape and texture coefficients are optimised. All the features used in Stage 4 and the specular highlight feature [11] are used at this stage. The cost function is:

$$
C_{5}=\omega_{1} C^{p}+\omega_{2} C^{c e}+\omega_{3} C^{t p}+\omega_{4} C^{s p}+\omega_{5} C^{s h}
$$

where $C^{s h}$ is the specular highlight feature.

\section{Experimental results}

\subsection{Experimental setup}

The PIE [15] database was used to evaluate the performance of the proposed method. PIE consists of face images of 68 subjects. Each person was imaged across 13 different



Figure 1. Fitting results. The first column shows the original input images from PIE. The second and third column are the fitted images without and with facial symmetry constraint, respectively.

poses, under 24 different illumination conditions ( 2 ambient light and 22 directed light variations) and with 4 different expressions. As this work focuses on illumination optimisation, we chose the illumination variation subset of the PIE database for experimentation. In order to avoid any bias caused by the pose and expression, we confine our experiments to the frontal pose and neutral expression images only. Specifically, the images of 68 subjects indexed by pose 27 (frontal pose) and illumination 2-22 are used. Illumination 1 and 23 were not used because they contain no directed light, only ambient light.

\subsection{Face reconstruction results}

By virtue of the 3D morphable model fitting, the shape and texture coefficients, rigid transform and illumination parameters are recovered. With these recovered parameters, the face can be reconstructed. The similarity of the input image and the reconstructed image is used to measure the fitting performance. Before the quantitative results are presented, some fitting examples are first shown in Fig.1.

The two input images are from illumination 02, which is the strongest side illumination in PIE, and therefore the most difficult illumination state for fitting. Since the facial symmetry constraint is introduced to mainly solve asymmetric illumination, the strong side illumination is a good example to show the advantage of the proposed method. Clearly, the illumination of the fitted images with facial symmetry constraint (3rd column) are better than without facial symmetry feature ( 2 nd column). The illumination of the 2nd column was under-estimated, because the fitting was trapped in a local minimum. This is the problem of most fitting strategies. Note also that the facial texture looks very noisy, because the texture coefficients are over- 


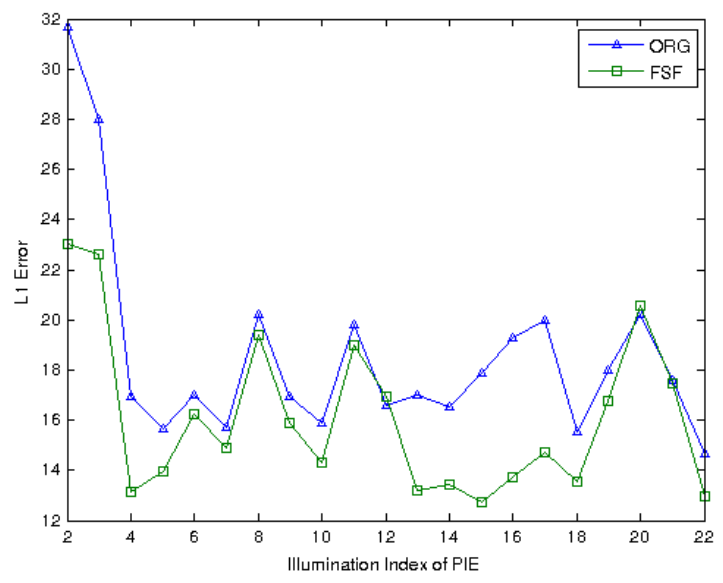

Figure 2. Face reconstruction performance. 'ORG' means original Multi-Feature fitting (MFF) strategy, and 'FSF' means fitting with facial symmetry constraint.

estimated. The 3rd column looks very natural and much smoother. It is clear that the illumination on the right part of the face was well recovered.

The quantitative results in Fig. 2 are presented in order to measure the face reconstruction performance in an objective way. We use the 11-norm to estimate the fitting error.

$e r r=\frac{1}{N} \sum_{i=1}^{N} \frac{1}{3}\left(\left|R_{i 1}-R_{i 2}\right|+\left|G_{i 1}-G_{i 2}\right|+\left|B_{i 1}-B_{i 2}\right|\right)$

where $R_{i 1}, G_{i 1}, B_{i 1}$ are the RGB values of the input image, $R_{i 2}, G_{i 2}, B_{i 2}$ are the RGB values of fitted image, and $N$ is the number of facial pixels. The greater err, the worse is the fitting.

From Fig.2, the curve produced by the method exploiting the facial symmetry constraint is more stable than that of the original method. It means the facial symmetry constraint makes the fitting process more robust to illumination variations. It is clear that the reconstruction error obtained with the proposed method is much smaller than that of the original method. In other words the facial symmetry constraint improved the face reconstruction performance significantly. The constraint helped to avoid local minima during the fitting process and consequently made it possible to recover better illumination parameters. In particular, the facial symmetry constrained worked much better at illumination states indexed 2,3,4,13,14,15,16,17 and 18. In those states, a strong side point light source casts asymmetric light on the face. The facial symmetry constraint helps to constrain the fitting process to avoid the texture coefficients being over-fitted. The worst reconstruction for both methods happened at illumination 02 , because it is the strongest side illumination (see samples of Fig.1). Even for our proposed method, the reconstruction error is still very high. The saturated illumination (highlighted parts in Fig.1) on the face image clipped the grey level values of the input image. Actually, what the model fitted is not the true signal but the clipped signal. This explains the bad reconstructions at illumination 2 for both methods. Figure 2 also shows that the reconstruction errors of both methods at illumination $8,11,21$ are almost the same. Point light sources of illumination 8,11 and 21 are near the centre of the face, so the directed light on the face is roughly symmetrical. In those three cases, the facial symmetry feature does not help much.

\subsection{Face recognition results}

After the fitting, the shape $(\alpha)$ and texture $(\beta)$ parameters are extracted from the input image. $\alpha$ and $\beta$ are actually the identity parameters because the non-identity parameters such as the pose and illumination parameters have been estimated and then removed. So face recognition task can be performed by comparing these identity parameters of the probe image and the gallery images. [2,11] concatenated the global and segmented $\alpha$ and $\beta$ normalised by their standard deviations to construct a vector $\mathbf{c}$

$$
\begin{aligned}
\mathbf{c}= & {\left[\frac{\alpha_{1}^{g}}{\sigma_{S, 1}}, \ldots, \frac{\alpha_{n_{\alpha}}^{g}}{\sigma_{S, n_{\alpha}}}, \ldots, \frac{\alpha_{1}^{s_{1}}}{\sigma_{S, 1}}, \ldots, \frac{\alpha_{n_{\alpha}}^{s_{1}}}{\sigma_{S, n_{\alpha}}}, \ldots \frac{\alpha_{n_{\alpha}}^{s_{4}}}{\sigma_{S, n_{\alpha}}},\right.} \\
& \left.\frac{\beta_{1}^{g}}{\sigma_{T, 1}}, \ldots, \frac{\beta_{n_{\beta}}^{g}}{\sigma_{T, n_{\beta}}}, \ldots, \frac{\beta_{1}^{s_{1}}}{\sigma_{T, 1}}, \ldots, \frac{\beta_{n_{\beta}}^{s_{1}}}{\sigma_{T, n_{\beta}}}, \ldots \frac{\beta_{n_{\beta}}^{s_{4}}}{\sigma_{T, n_{\beta}}}\right]
\end{aligned}
$$

where superscript $g$ denotes the global model and superscripts $s_{1}$ to $s_{4}$ denote the 4 segments (eyes, nose, mouth and the rest) of the model, $\sigma_{S, i}$ with $\sigma_{T, i}$ denote the standard deviation of the $i$ th shape and texture parameters respectively. $n_{\alpha}$ and $n_{\beta}$ denote the number of $\alpha$ and $\beta$ parameters, respectively.

After $\mathbf{c}$ is constructed, the similarity should be defined to measure the distance between two such vectors. [2] proposed a method based on a Maximum-Likelihood classifier and Linear Discriminant Analysis, aiming to remove the residual errors of model fitting. The method has to be trained using a training set. A much simpler solution was proposed in [11] who just used the angle between the two vectors to measure similarity. This method does not require any training. We have adopted this approach in our work, i.e. the similarity of two images is measured as

$$
d=\frac{c_{1}^{T} \cdot c_{2}}{\sqrt{\left(c_{1}^{T} \cdot c_{1}\right)\left(c_{2}^{T} \cdot c_{2}\right)}}
$$

In the experiment we conducted, the images of illumination 01 were designated as the gallery images because they were captured in ambient light. None of these images are affected by directed light, so they should extract the facial features correctly. The probe images are all images with the directed light switched on (illumination 2-22). Fig.3 shows the results of face recognition. 


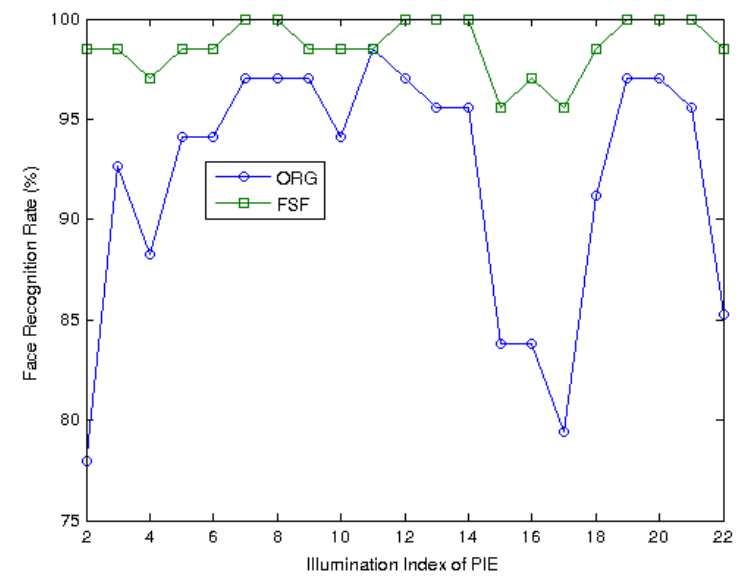

Figure 3. Face recognition performance. 'ORG' means original Multi-Feature fitting (MFF) strategy, and 'FSF' means fitting with facial symmetry constraint.

From Fig.3, it is clear that the face recognition rate of our proposed method is higher than that of the original MFF method at all the illumination variations. Thus our proposed method works consistently better than the original MFF method. The proposed method achieved a very high face recognition rate for all illuminations.

Of particular interest is the illumination variations where a strong side illumination is present, eg. illuminations 2,3 , 20 , and 21 . In all these cases, the recognition rate was considerably improved by our proposed approach due to better recovery of facial texture in presence of strong side illumination.

\section{Conclusions and future work}

The fitting of the 3D morphable model is an ill-posed problem. This problem has been approached by a number of researchers. One of its aspects, the problem of recovering the illumination, is particularly challenging. Although different optimisation strategies have been proposed in the literature to deal with this problem, a comprehensive solution still remains elusive. In this work, we proposed the use of a prior in the form of facial symmetry constraint which is shown to produce better illumination estimate, and as a result improved fitting performance. The constraint is incorporated into a 5-stage multi-feature optimisation framework. The experimental results obtained on the PIE database show the merit of the proposed method as assessed in terms of two different criteria: face reconstruction error and face recognition rate. Both have been improved significantly.

In the future, we will extend the proposed method to face recognition with pose variations.
Acknowledgement This work was carried out as part of EPSRC project ACASVA (Adaptive cognition for automated sports video annotation) under contract $\mathrm{EP} / \mathrm{F} 069421 / 1$. The EPSRC financial support is gratefully acknowledged.

\section{References}

[1] V. Blanz and T. Vetter. A morphable model for the synthesis of 3D faces. In Proceedings of the 26th annual conference on Computer graphics and interactive techniques, pages 187194, 1999.

[2] V. Blanz and T. Vetter. Face recognition based on fitting a 3D morphable model. Pattern Analysis and Machine Intelligence, IEEE Transactions on, 25(9):1063-1074, 2003.

[3] T. Cootes, G. Edwards, and C. Taylor. Active appearance models. Pattern Analysis and Machine Intelligence, IEEE Transactions on, 23(6):681-685, 2001.

[4] G. Hu, C. Chan, J. Kittler, and W. Christmas. Resolutionaware 3D morphable model. In British Machine Vision Conference, 2012.

[5] B. Kang, H. Byun, and D. Kim. Multi-resolution 3D morphable models and its matching method. In Pattern Recognition, 19th International Conference on, pages 1-4. IEEE, 2008.

[6] Y. Liu and J. Palmer. A quantified study of facial asymmetry in 3d faces. Technical Report, 2003.

[7] I. Matthews and S. Baker. Active appearance models revisited. International Journal of Computer Vision, 60(2):135164, 2004.

[8] P. Mortazavian, J. Kittler, and W. Christmas. 3D morphable model fitting for low-resolution facial images. In Biometrics, 5th IAPR International Conference on, pages 132-138. IEEE, 2012.

[9] G. Passalis, P. Perakis, T. Theoharis, and I. Kakadiaris. Using facial symmetry to handle pose variations in real-world $3 \mathrm{~d}$ face recognition. Pattern Analysis and Machine Intelligence, IEEE Transactions on, 33(10):1938-1951, 2011.

[10] J. T. Rodriguez. 3D Face Modelling for 2D+3D Face Recognition. PhD thesis, Surrey University, Guildford, UK, 2007.

[11] S. Romdhani. Face image analysis using a multiple features fitting strategy. $\mathrm{PhD}$ thesis, University of Basel, Switzerland, 2005.

[12] S. Romdhani, V. Blanz, and T. Vetter. Face identification by fitting a $3 \mathrm{~d}$ morphable model using linear shape and texture error functions. ECCV 2002, pages 3-19, 2006.

[13] S. Romdhani and T. Vetter. Efficient, robust and accurate fitting of a 3D morphable model. In Computer Vision. Proceedings. 9th IEEE International Conference on, pages 5966. IEEE, 2003.

[14] E. Saber and A. Tekalp. Frontal-view face detection and facial feature extraction using color, shape and symmetry based cost functions. Pattern Recognition Letters, 19(8):669-680, 1998.

[15] T. Sim, S. Baker, and M. Bsat. The cmu pose, illumination, and expression (pie) database. In Automatic Face and Gesture Recognition, 2002. Proceedings. 5th IEEE International Conference on, pages 46-51, 2002. 